\title{
CHANGEMENTS D'ÉTATS EN CONCEPTION ARCHITECTURALE : RÉMANENCE, RÉSISTANCE, RÉSILIENCE
}

\author{
Author(s) / Auteur(s) : \\ Damien CLAEYS \\ Professeur \\ Faculté d'architecture, d'ingénierie architecturale, d'urbanisme (Loci) \\ Laboratoire Théorie des systèmes en architecture (tsa-lab) \\ Université catholique de Louvain (UCLouvain) \\ damien.claeys@uclouvain.be
}

\begin{abstract}
Résumé :
Dans le présent texte spéculatif, trois postulats sont posés dans le cadre de l'explicitation des processus de conception de projets d'architecture. Premièrement, la conscience du concepteur est modélisable à partir d'une posture émergentiste. Deuxièmement, tout processus mental de conception est modélisable comme un système dynamique. Troisièmement, tout image mentale du projet d'architecture est modélisable d'un point de vue, à la fois, perceptif, statique et dynamique. De ces trois postulats, dans tout processus de conception architecturale, lorsqu'une image mentale du projet d'architecture évolue d'un état à un autre, le concepteur utilise des opérations de conception (composition, construction et régulation) pour actualiser un modèle architectural dont le degré de persistance est variable. Par analogie, les trois concepts de rémanence, résistance et résilience n'apporteraientils pas un éclairage sur ces changements d'état du modèle architectural, en dialoguant avec le concept général d'équilibre? La rémanence perceptive, la résistance statique et la résilience dynamique expriment les modalités de persistance du modèle mental du projet d'architecture, face aux opérations de composition, de construction et de régulation.
\end{abstract}

\section{Keywords / Mots-clés :}

rémanence, résistance, résilience, architecture, conception, modélisation, heuristique, processus, opération, opérance, persistance, intégrité, équilibre, système, structure, ensemble, composition, construction, régulation, élasticité, tenségrité, plasticité

Pour commencer, quelques précautions oratoires doivent être prises.

Premièrement, de nombreuses théories placent le champ de la recherche en architecture dans les édifices ou - au sens large - dans l'étude des multiples artefacts, en projet ou matérialisés, présents dans l'environnement construit (par exemple, des traités classiques définissent des règles de composition esthétiques, des doctrines postmodernistes établissent des typologies...). En adoptant une posture méta par rapport à ce type d'approches, la théorie de la conception partiellement construite ici place ce champ directement dans les processus menés par les concepteurs ${ }^{1}$, en partant du principe que l'architecture n'a de réelle existence que dans les consciences des concepteurs qui conçoivent les édifices et des usagers qui les habitent. Deuxièmement, le présent texte est un essai spéculatif ayant pour finalité d'enrichir un modèle heuristique ${ }^{2}$ de la conception architecturale développé dans d'autres écrits auxquels il sera les

\footnotetext{
1 Le mot concepteur est utilisé ici dans un sens large, il peut désigner une femme ou un homme, mais également une machine dotée d'intelligence artificielle ou toute hybridation entre ces différents êtres.

2 Le modèle est un raisonnement heuristique dans le sens où il est construit pour favoriser la découverte d'une théorie à partir de connaissances incomplètes du phénomène étudié - la conception architecturale - de manière à aboutir, dans un temps raisonnable, à des conclusions vraisemblables, bien que sous-optimales (Le Moigne, 1990). Le formalisme mathématique est utilisé ici pour exprimer avec précision et concision des concepts difficilement exprimables avec des mots, mais aucune correspondance directe n'existe entre ces expressions logiques et des phénomènes réels, c'est le principe de toute modélisation.
} 
conditions externes affectant le travail du concepteur sont volontairement omises, ce qui ne minimise en rien leur importance ${ }^{3}$.

Pour contextualiser le propos développé ici, trois postulats sont posés.

\section{LE CONCEPTEUR : UNE POSTURE ÉMERGENTISTE}

Sachant qu'aucun processus de conception architecturale ne puisse être étudié sans tenir compte de l'activité cognitive de celle ou celui qui le conçoit, le premier postulat consiste à dire que la conscience d'un concepteur de projets d'architecture est modélisable à partir d'une posture émergentiste.

En partant de l'hypothèse que la réalité est une construction mentale et qu' "il n'existe pas de réalité absolue, mais seulement des conceptions subjectives et souvent contradictoires de la réalité" (Watzlawick, 1978), la réalité n'est pas le réel et elle n'est pas non plus unique. Une réalité différente se trouve dans chaque conscience individuelle et chacune d'elles évolue dynamiquement en fonction de la personnalité et des expériences de celui qui la pense. Si bien que : "La distinction entre le réel et les réalités est inhérente à la condition humaine" (Claeys, 2018).

Le filtre de la perception et la coupure du langage mènent l'être humain à donner naissance à un monde imaginaire, "un double du réel co-construit par l'homme - qualifié d'augmenté - contre lequel, impertinent, le réel résiste dès qu'il est provoqué" (Claeys, 2013). Ainsi, le concept de "réel augmenté" permet d'opérer une distinction entre les réalités construites et le réel. La réalité augmentée $(A R)$ - semiimmersive, en ajoutant des objets virtuels dans un environnement réel - et la réalité virtuelle $(V R)-$ immersive, en créant virtuellement un environnement réel - nécessitent des moyens techniques et matériels dans le réel pour surimposer à ce dernier des informations. La $A R$ et la $V R$ produisent des stimuli potentiels capables d'exciter les capteurs externes du corps, pour compléter la perception du monde de l'usager. Au contraire, "le réel augmenté $R^{\prime}$ est intrapsychique, produit naturellement par la conscience" (Claeys, 2017b) ${ }^{4}$.

De manière heuristique, à partir de son interaction créative avec l'environnement, l'être humain peut être représenté par une boucle dynamique, ancrée dans le réel $R$, à partir de laquelle émerge une seconde boucle dynamique, génératrice du réel augmenté $R^{\prime}$ (Claeys, 2017b) ${ }^{5}$. Autrement dit, il peut être représenté par une première boucle au niveau physiologique (stimulation/action), d'où émerge une deuxième boucle au niveau cognitif (interprétation/projection). La première étant nécessaire à l'émergence de la seconde, mais pas suffisante ( $c f$. figure 1). Ce modèle est non dualiste dans le sens où il ne repose pas sur l'existence de deux instances séparées : un corps et une âme. L'âme n'est pas extérieure et détachable du corps, mais l'être humain possède une liberté de penser. Selon le philosophe des sciences belge Bernard Feltz (2003), avec une posture émergentiste, cette liberté peut être pensée comme "un espace d'indétermination, propriété de l'organisme humain, non réduite aux propriétés des éléments constituants, non réduite à sa structure biologique". Il explique que la position émergentiste présente une causalité descendante (downward causation) : le niveau supérieur de la conscience possède une autonomie propre, mais il possède avec le niveau inférieur du corps un rapport de condition de possibilité. Selon lui, l'être humain n'est pas libre physiologiquement, mais la liberté est pensable comme une capacité d'autodétermination : le libre arbitre est la "possibilité de choix entre diverses alternatives dans le comportement humain".

L'apparente liberté de l'être humain s'exprime dans l'autonomie avec laquelle il construit son réel augmenté $R^{\prime}$ à partir d'élans affectifs, de recherches spirituelles, d'émerveillements esthétiques, de la hiérarchisation qu'il donne à ses besoins physiologiques, psychologiques et sociaux. Le réel augmenté

\footnotetext{
3 Les facteurs externes au processus sont nombreux et mériteraient d'être également étudiés : nombre de co-concepteurs, rythme spatiotemporel dans lequel le concepteur travaille, limites budgétaires du projet, ergonomie du lieu de la conception, état d'esprit, fatigue et agilité cognitive du concepteur... Le mot conception sous-entend ici la possibilité de la co-conception.

${ }^{4}$ Bien que la réalité virtuelle soit toujours présentée comme immersive, dans l'absolu, elle ne pourra jamais l'être totalement puisqu'elle est incapable de créer le réel.

5 Dans des travaux précédents, un plus distinguait $R^{+}$de $R$. Mais le réel augmenté est, à la fois, moins que le réel, puisque notre conscience souffre d'une limite cognitive dans le traitement des informations extérieures et, plus que le réel, puisque notre conscience est capable de faire émerger un monde intérieur qui n'existe pas dans le réel. Un prime est donc utilisé pour le distinguer du réel.
} 
peut être considéré comme une propriété émergente de la structure biologique complexe caractérisant l'être humain qui le construit ${ }^{6}$.

La première boucle est l'expression d'un substrat biologique - par analogie, un hardware - qui fonctionne par "computation", alors que la seconde boucle est la représentation d'une émergence cognitive - par analogie, un software - qui fonctionne par "cogitation" (Morin, 1986) ${ }^{7}$ :

- la première boucle est à l'œuvre entre l'environnement et le corps biologique de l'être humain. Les stimuli physiques - les inputs - viennent, à la fois, de l'extérieur (du contexte) et de l'intérieur (du corps). Les stimuli excitent les récepteurs des organes sensoriels, les capteurs extérieurs (yeux, oreilles, peau, langue, nez) et internes (sensibilité viscérale des organes, surface cutanée, proprioception des articulations et des muscles) en variant l'état des nerfs sensoriels ${ }^{8}$. À partir d'un traitement de l'information brute, le corps produit une synesthésie des sens pour construire une sensation psychologique ${ }^{9}$;

- la seconde boucle est active entre l'image mentale que l'être humain construit de son environnement et celle qu'il possède de son propre corps en relation avec cet environnement. Le cerveau décode la sensation par un processus de perception externe et interne (la perception rend intelligible la sensation en identifiant une signification à la source de celle-ci) et il opère une synthèse des informations parfois contradictoires, archivées dans ses mémoires et décodées par la perception, avant de concevoir ses actions futures.

De l'établissement de ces deux boucles, l'être humain est donc "un être (auto)organisé en interaction dynamique avec son environnement" qui adapte son comportement en fonction d'un "projet téléologique", une "finalité projective interne" qu'il poursuit à court/moyen/long terme (Claeys, 2016). Un projet téléologique tel que décrit ici est "le catalyseur d'un processus d'action menant à une finalité interne (celle fixée par le système lui-même)". Ce type doit clairement être distingué d' "une programmation téléonomique associée à une finalité externe (celle du modélisateur du système)" ou d' "une destinée théologique sous l'effet d'une cause extérieure (celle d'une intelligence divine)" (Claeys, 2016). L'être peut donc être considéré comme un agens (agissant) dans le sens où cet être (auto)organisé est une entité qui agit en fonction d'un projet personnel. Cette finalité subjective, dont les motivations varient, est capable d'orienter des stratégies d'action pour répondre à des besoins physiologiques, psychologiques et sociaux.

En évitant les deux postures extrêmes, celle du "solipsisme" du côté du "sujet qui connaît", niant l'existence d'un réel extérieur à la conscience, et celle du "réalisme naïf" du côté de la "chose à connaître", niant l'existence de toute forme de subjectivité dans l'appréhension du réel" (Steiwer, 2010), la posture émergentiste positionne le champ d'étude des processus de conception architecturale dans le réel augmenté, dans un espace de conception mental, acceptant la subjectivité inhérente aux actes de pensée du concepteur.

\footnotetext{
${ }^{6}$ La posture n'est pas réductionniste non plus dans le sens où la conscience est bien le produit de l'évolution naturelle, mais son étude nécessite une approche différente de celle du corps.

7 Pour Edgar Morin (1986, pp.122-123), le cogito ergo sum cartésien doit être complété en un cogito ergo computo ergo sum, pour montrer l'existence de deux niveaux logiques dialogiques (qui ne peuvent exister l'un sans l'autre) : le computo-cerveau (présent chez tous les êtres vivants) et le cogito - esprit (présent chez les humains).

8 Chaque sens est associé à un organe sensoriel, sensible à des sollicitations différentes : des ondes lumineuses frappent notre rétine, des ondes sonores frappent nos tympans, des molécules stimulent notre langue et nos narines. La peau est soumise à un grand nombre de sollicitations variables : des stimuli externes, à la fois, physiques (thermique, mécanique, électrique, rayonnement) et chimiques, ainsi que des stimuli indirects (chocs, inflammations, variations de la circulation sanguine, stress émotionnels...).

9 Le mot synesthésie venant du latin syn- (avec, union) et aesthesis (sensation) est utilisé ici par analogie pour exprimer en un mot le croisement des cinq sens. Mais la vraie synesthésie est neurologique et involontaire. Elle vient des récepteurs sensoriels qui réagissent à une excitation venue de l'extérieur du corps biologique.
} 


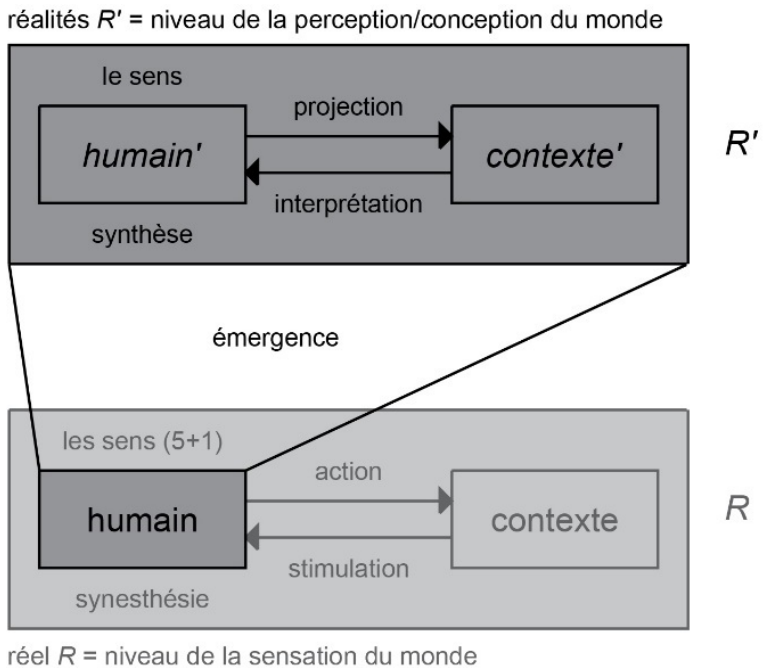

Fig. 1 - Damien Claeys, L'émergence dynamique de $R^{\prime}$ à partir de l'interaction du corps avec le contexte dans $R$.

\section{LE PROCESSUS DE CONCEPTION : UN SYSTÈME DYNAMIQUE}

Sachant que tout concepteur possède une connaissance subjective des données du projet d'architecture à concevoir, le second postulat consiste à dire que tout processus de conception est modélisable comme un système dynamique.

À défaut de pouvoir être complètement définie, la conception architecturale est un phénomène complexe modélisable heuristiquement. Pour produire un projet d'architecture menant à la construction potentielle d'un artefact architecturé, un concepteur initie "un processus de conception au cours duquel il opère mentalement à plusieurs reprises sur un modèle jusqu'à proposer une solution satisfaisante parmi d'autres possibles" (Claeys, 2015). À défaut d'être linéaire, ce processus est plutôt "circulaire", "itératif" et "réflexif", passant alternativement par des phases de "convergence" et de "divergence" avant d'aboutir à une "solution sous-optimale parmi d'autres" (Claeys, 2013). Le concepteur oriente le processus de conception à la manière d'un "statisticien" intégrant une "dimension probabiliste" (Claeys, 2017b), en fonction d'une "finalité projective" (Claeys, 2016).

Le principal processus animant le déroulement de la conception est représenté par une succession d'états discrets, actualisés par des opérations cognitives ${ }^{10}$. Chaque état successif est une image mentale du projet d'architecture en cours de conception. Chaque image constitue une occurrence du modèle $\operatorname{architectural}\left(M_{\mathrm{i}}\right)$. Autrement dit, elles figurent des occurrences de la représentation dynamique du projet dans la conscience du concepteur. Les états sont indexés à partir des valeurs prises par un indice $i$, attribué chronologiquement.

De là, $\forall i \in\{0,1,2,3, \ldots, k, \ldots, n\}$, tout processus démarre à un état 0 , passe par un état $k$ donné et se termine à un état $n$. Si un modèle architectural $M_{\mathrm{k}}$ est une image mentale à un instant donné $k$ du projet d'architecture en cours de conception et si une opération architecturale $O_{\mathrm{k} \rightarrow \mathrm{k}+1}$ est un acte cognitif capable d'actualiser ce modèle d'un état $k$ à un état $k+1$, alors un processus de conception est modélisable heuristiquement comme un système dynamique, en tant que succession non-linéaire d'occurrences du modèle $M_{\mathrm{i}}$, rythmées par des opérations $O_{\mathrm{i} \rightarrow \mathrm{i}+1}$ et orientée en fonction de la finalité projective du concepteur (Claeys, 2013 ; Claeys, 2015).

\footnotetext{
${ }^{10}$ Deux autres processus en interaction répétée avec celui des images mentales ne sont pas pris en compte ici, même s'ils restent importants : "la succession des états de la mémoire active et des actualisations/conservations opérées entre eux" et "la succession des perceptions actives des médias externes (plans, schémas, dessins, gestuelles, animations... ou tout autre forme de média observable dans le réel $R$ )" (Claeys, 2017b).
} 


\section{LE MODÈLE MENTAL : PERCEPTIF, STATIQUE, DYNAMIQUE}

Le troisième postulat consiste à dire que toute image mentale du projet d'architecture est modélisable d'un point de vue, à la fois, perceptif, statique et dynamique.

À la différence du réel $R$, le réel augmenté $R^{\prime}$ est structuré par le concepteur à l'aide, au minimum, de trois "niveaux d'organisations" (configuration perceptive ${ }^{11}$, "structuralisme hiérarchique", "émergentisme systémique"), liés à une triade de concepts étudiés en sciences (forme, structure, fonction $)^{12}$. Parallèlement à ces trois niveaux d'organisation, trois "constructions élémentaires" permettent d'opérer dans le réel augmenté : l'ensemble, la structure, le système (Claeys, 2013).

Autrement dit, le concepteur modélise dans sa tête à partir des :

- ensemble : "toute collection d'éléments, réels ou non, (re)groupés en fonction d'un caractère commun [...]. La construction mentale d'un ensemble précède toute construction éventuelle d'une structure ou d'un système" (Claeys, 2013);

- structure : "tout ensemble d'éléments, réels ou non, en interaction statique et organisé hiérarchiquement [...]. La construction mentale d'une structure présuppose l'existence d'un ensemble et précède toute construction éventuelle d'un système" (Claeys, 2013);

- système : "un ensemble d'éléments en interaction dynamique orientés en fonction d'un but" (Rosnay, 1975). "La construction mentale d'un système présuppose l'existence d'une structure et donc également d'un ensemble" (Claeys, 2013).

L'existence de ces trois constructions élémentaires rend possible pour le concepteur d'organiser le modèle architectural $M_{\mathrm{i}}$ à partir de plusieurs points de vue (perceptif ${ }^{13}$, statique et dynamique), si bien qu'il peut mener trois types d'opérations sur les trois plans du modèle mental du projet d'architecture :

- l'opération perceptive de composition $O_{1, \mathrm{i} \rightarrow \mathrm{i}+1}$ sur la plan perceptif $M_{1, \mathrm{i}}$ du modèle architectural modélisé à l'aide d'ensembles ;

- l'opération analytique de construction $O_{2, \mathrm{i} \rightarrow \mathrm{i}+1}$ sur la plan statique $M_{2, \mathrm{i}}$ du modèle architectural modélisé à l'aide de structures ;

- l'opération systémique de régulation $O_{3, \mathrm{i} \rightarrow \mathrm{i}+1}$ sur la plan dynamique $M_{3, \mathrm{i}}$ du modèle architectural modélisé à l'aide de systèmes.

En fonction des "espaces de références" auxquels il se réfère et de l' "espace de conception" dans lequel il opère, tout concepteur modélise donc les images mentales successives de son projet d'architecture en choisissant l'une ou en combinant les modalités perceptive, statique ou dynamique des occurrences du modèle architectural sur lesquelles il opère.

Chaque opération cognitive menée par le concepteur entre deux états successifs du processus de conception architecturale peut donc être formalisée heuristiquement sous la forme d'une fonction égale à la combinaison linéaire - une expression construite à partir de la somme de plusieurs termes multipliés par une constante - dont les termes reprennent les trois plans du modèle architectural.

De là, $\forall i \in\{0,1,2,3, \ldots, k, \ldots, n\}$, toute opération générale de conception $O_{\mathrm{i} \rightarrow \mathrm{i}+1}$ est la combinaison linéaire des opérations particulières de composition $O_{1, \mathrm{i} \rightarrow \mathrm{i}+1}$, de construction $O_{2, \mathrm{i} \rightarrow \mathrm{i}+1}$ et de régulation $O_{3, \mathrm{i} \rightarrow \mathrm{i}+1}$, pondérée par des poids $a, b$ et $c$, et menées sur les plans perceptif $M_{1, \mathrm{i}}$, statique $M_{2, \mathrm{i}}$ et dynamique $M_{3, \mathrm{i}}$ du modèle architectural $M_{\mathrm{i}}$. Cette opération produit une nouvelle occurrence actualisée du modèle architectural $M_{\mathrm{i}+1}$, obtenue après un changement $C h_{\mathrm{i} \rightarrow \mathrm{i}+1}$. Ce qui donne, à un instant $k$ donné, l'équation heuristique suivante :

$$
O_{\mathrm{k} \rightarrow \mathrm{k}+1}\left(M_{\mathrm{k}}\right)=\left(a \times O_{1, \mathrm{k} \rightarrow \mathrm{k}+1}\left(M_{1, \mathrm{k}}\right)+b \times O_{2, \mathrm{k} \rightarrow \mathrm{k}+1}\left(M_{2, \mathrm{k}}\right)+c \times O_{3, \mathrm{k} \rightarrow \mathrm{k}+1}\left(M_{3, \mathrm{k}}\right) \Rightarrow C h_{\mathrm{k} \rightarrow \mathrm{k}+1}\right.
$$

\footnotetext{
${ }^{11}$ Dans des travaux précédents, le premier niveau d'organisation était celui de la "configuration formelle" (Claeys, 2013), mais il est appelé ici la configuration perceptive pour éviter toute connotation esthétisante ou plastique.

${ }^{12}$ Par ailleurs, un parallèle entre le développement de ces concepts dans l'histoire des sciences et ceux de la triade vitruvienne (venustas, firmitas, utilitas) en histoire et théorie d'architecture montre la richesse des échanges conceptuels possibles entre l'architecture et d'autres champs disciplinaires (Claeys, 2013).

${ }^{13}$ Dans des travaux précédents, l'expression "modèle agrégatif" était utilisée (Claeys, 2013), mais elle n'était pas assez explicite.
} 


\section{ÉCHANTILLONNAGE ET PERSISTANCE PARTIELLE DU MODÈLE MENTAL}

Tout en rappelant qu'il est terriblement plus perfectionné et complexe qu'un cinématographe, le neurologue français Lionel Naccache (2020) décrit - par analogie - notre système cognitif comme une forme de "cinéma intérieur". En effet, l'analogie entre le cinématographe et le fonctionnement de notre cognition est particulièrement éclairante en se rappelant que la magie du cinéma fonctionne à l'aide de la projection de 24 images par seconde sur un écran.

Sans que ce mécanisme soit conscientisé par le sujet connaissant, le substrat biologique du système neuronal génère une forme d'échantillonnage, réduisant le signal continu des informations transmises par les organes perceptifs à une suite d'images fixes, avec une fréquence d'environ 13 images par seconde ${ }^{14}$. Et ce fait ne concerne pas que la perception. De manière générale, la conscience fonctionne au rythme d'un "staccato constitué d'une succession d'états discrets que nous percevons comme un flux mental continu" (Naccache, 2020).

Inconsciemment, au cours du processus, le concepteur discrétise le flux continu des pensées traversant sa conscience en une série d'occurrences remarquables du modèle mental du projet d'architecture qu'il conçoit (Claeys, 2020). Autrement dit, sa conscience génère automatiquement une forme d'échantillonnage du flux continu de ses pensées, elle réduit un processus continu à une suite d'images mentales fixes, dont chacune capture un état donné du projet en cours.

En détectant des similitudes entre plusieurs occurrences successives, le concepteur identifie une forme d'intégrité $e^{15}$ à la représentation mentale du projet qu'il imagine (par exemple, la détection d'une figure sur un fond, des limites du modèle architectural, un schème...). Mais cette intégrité temporaire est celle d'une représentation interne en devenir, les actes de pensée se succèdent inexorablement, rendant illusoire la consistance conférée au projet. Même si le concepteur attribue une intégrité particulière à une séquence de représentations internes du projet, le dynamisme ininterrompu du flux des images mentales le portera à la faire évoluer ${ }^{16}$.

D'un état à l'autre, réévaluant son intégrité partielle, le concepteur fixe l'organisation de certaines parties du modèle architectural, tout en autorisant l'évolution d'autres. D'une occurrence à l'autre, après un changement $\left(C h_{\mathrm{k} \rightarrow \mathrm{k}+1}\right)$, consécutif à une opération de conception $\left(O_{\mathrm{k} \rightarrow \mathrm{k}+1}\right)$, le concepteur ayant attribué une intégrité au modèle architectural à un instant $k\left(M_{\mathrm{k}}\right)$ peut en attribuer une autre à un instant $k+1$ $\left(M_{\mathrm{k}+1}\right)$. L'évolution de l'intégrité du modèle revient à attribuer une capacité de persistance ${ }^{17}$ à l'image mentale du projet.

Parmi d'autres possibles, trois concepts transdisciplinaires déclinent la propriété de persistance du modèle architectural respectivement du point de vue de ses trois plans perceptif, statique et évolutive : la rémanence, la résistance et la résilience.

\footnotetext{
${ }^{14}$ En provoquant expérimentalement l'illusion de la roue qui tourne à l'envers (l'illusion du mouvement inversé), une première équipe de chercheurs (Purves et al., 1996) a démontré que, de manière générale, la conscience construit ses perceptions par le prisme d'un échantillonnage discret de la scène visuelle et elle a estimé sommairement la fréquence de cette discrétisation entre 2 et 20 images par seconde. Plus récemment, une autre équipe (VanRullen et al., 2006) a provoqué expérimentalement la même expérience, mais en enregistrant l'activité cérébrale des participants à l'aide d'un électroencéphalographe, ce qui lui a permis de déterminer que la fréquence de la projection sur la "scène de notre conscience" (Naccache, 2020) est d'environ 13 images par secondes.

${ }^{15}$ Composé du verbe tango (toucher) et du préfixe négatif in, les mots latins integer (intact, entier, non touché, non entamé) et integritas (intégrité, chasteté, virginité) ont donné le mot intégrité signifiant, à la fois, l'état d'une chose qui est dans son entier ou celui d'un être dénué de mauvaises intentions.

${ }^{16}$ Cette précision est particulièrement importante lorsque le concepteur modélise un projet sous la forme d'une "construction élémentaire" de type structure dont la définition affirme clairement le caractère statique et hiérarchique. Les structures décrites ici ne désignent pas uniquement des modèles de structures mécaniques assurant la stabilité de l'édifice projeté. Le processus dynamique de conception permet le passage d'un état statique à l'autre du modèle architectural.

${ }^{17}$ Le mot persistance vient du latin persistere dérivé du verbe sistere (se tenir debout, construire, soutenir, empêcher de tomber) avec le préfixe per- (durant, à travers le temps).
} 


\section{LA RÉMANENCE DU MODÈLE PERCEPTIF}

Le mot rémanence ${ }^{18}$ est sans doute moins utilisé que les mots résistance et résilience, pourtant il est important dans plusieurs disciplines.

La rémanence décrit différentes formes de persistance d'un effet, alors que la cause de son apparition a disparu : une légère traînée apparaissant à la suite d'un objet en mouvement rapide sur les écrans, la persistance des effets d'un pesticide dans l'environnement après son utilisation, de l'aimantation d'une substance ferromagnétique, coupée de l'effet de tout champ magnétique, de l'étanchéité des joints souples après la suppression des contraintes, des données numériques sur un support de stockage ou même de l'atmosphère imprégnant un lieu précis, après la tenue d'un événement historique.

Mais dans le cas de la conception architecturale où le concepteur crée constamment des images mentales, le sens du mot en psychologie est le plus pertinent.

En psychophysiologie, le mot rémanence évoque la propriété de certaines sensations de subsister après la disparition de l'excitation qui leur a donné naissance (par exemple, la rémanence des images visuelles lors d'un choc post-traumatique). Plus précisément, la rémanence est la propriété d'une sensation, notamment visuelle, de persister après la disparition du stimulus. $\mathrm{Au} \mathrm{XIX}{ }^{\mathrm{e}}$ siècle, le physicien belge Joseph A. F. Plateau est célèbre pour ses recherches sur la pertinence rétinienne qui lui ont notamment permis d'inventer le phénakistiscope ( $c f$. figure 2), capable de créer l'illusion d'un mouvement continu à partir d'une succession d'images. À cette époque, les scientifiques se focalisaient sur les propriétés intrinsèques des yeux, sur la persistance des images imprimées sur la rétine des yeux pour expliquer les illusions d'optique. Aujourd'hui, ils pensent plutôt que c'est la conscience qui crée subjectivement les images mentales créées à partir de la sollicitation de nos organes perceptifs. En effet, selon Lionel Naccache (2020), "à l'entrée de notre système perceptif, nos rétines reçoivent un flux continu d'informations lumineuses" et "le fruit de notre perception consciente est lui aussi structuré subjectivement comme un film continu", mais "il existe un mécanisme d'échantillonnage discret des images" entre ces deux processus continus. Il pose alors que notre perception visuelle enchaîne trois étapes : "continuité objective - discrétisation des images - continuité subjective perçue" ${ }^{19}$.

La rémanence doit être distinguée de la résurgence et de la réminiscence, désignant le fait qu'un phénomène déjà apparu puisse réapparaître, après une absence relativement longue du champ perceptif, de manière consciente ou inconsciente. Elle désigne la persistance partielle d'une représentation au cours d'une séquence constituée de plusieurs images mentales consécutives.
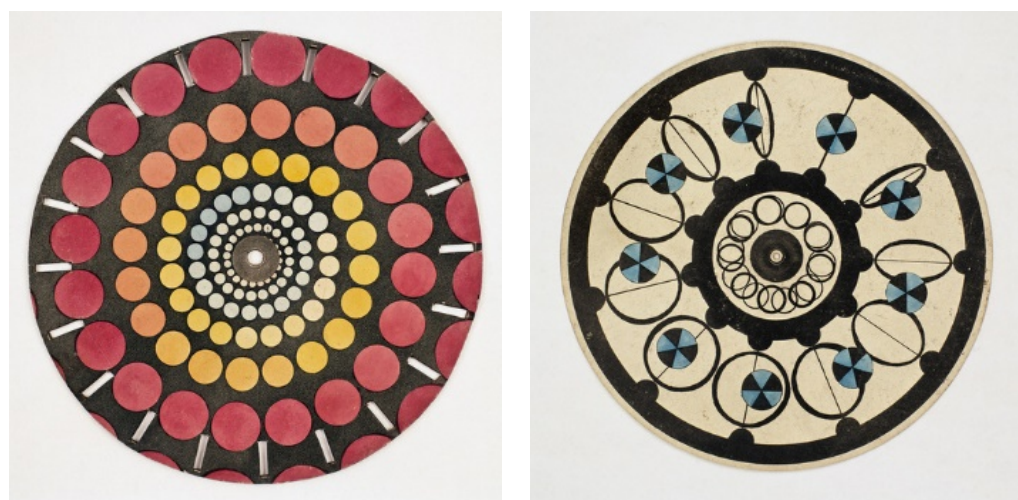

Fig. 2 - 1. Joseph Plateau, Disque en carton à vingt fentes obturatrices de phénakistiscope, lithographie en couleurs sur une face, 1833. 2. Alphonse Giroux, Disque en carton non fenestré de phénakistiscope, estampe rehaussée sur une face, 1833.

Collections de la cinémathèque française et du CNC.

\footnotetext{
${ }^{18}$ Le mot rémanence vient du verbe latin remanere (rester). Dès le $\mathrm{XII}^{\mathrm{e}}$ siècle, il désigne la demeure, la résidence, le fait de rester au même endroit, avant de désigner, au début du $\mathrm{XX}^{\mathrm{e}}$ siècle, la persistance d'un effet après que la cause ait été supprimée.

${ }^{19} \mathrm{La}$ détermination des différents réseaux cérébraux impliqués dans ces trois étapes fait encore l'objet de nombreuses recherches, dont les résultats sont parfois contradictoires.
} 
De ce qui précède, au cours d'un processus de conception architecturale, nous posons que la rémanence est le degré de persistance de la dimension perceptive du modèle architectural au cours d'une séquence d'images mentales du projet, la persistance partielle d'une représentation formelle du projet d'architecture au cours du temps.

\section{LA RÉSISTANCE DU MODÈLE STATIQUE}

Les mots résistance ${ }^{20}$ et résilience sont plus utilisés que le mot rémanence et ils sont régulièrement opposés.

En physique des matériaux, les concepts d'élasticité et de plasticité sont distingués : l'élasticité d'une matière lui permet de retrouver sa forme initiale après une déformation lorsque la sollicitation cesse (à l'image d'un élastique qui reprend sa forme une fois détendu), alors que la plasticité de la matière ne lui permet pas de retrouver sa forme initiale (bien qu'elle puisse posséder une certaine résistance à la déformation, lui donnant un degré de malléabilité). En poussant peut-être un peu loin l'analogie, nous pourrions dire que l'élasticité d'un matériau lui donne une capacité de résistance, alors que la plasticité lui confère une forme de résilience.

La résistance est la capacité à faire obstacle à quelque chose au sens propre ou figuré : résister à ses passions, résister pour ne pas être détruit par une action extérieure (par exemple, résister à la chaleur, résister à une catastrophe naturelle...).

En philosophie, la résistance est l'une des qualités premières des corps, celle que possède la matière sensible d'être perceptible au toucher, à l'effort musculaire. Ce qui résiste hors de nous, c'est une chose à laquelle nous attribuons une substance. Elle est la possibilité pour un phénomène de prouver son existence en soi. La sensation de résistance est la consolidation directe de l'existence, de ce qui résiste.

En psychanalyse, la résistance est ce qui fait obstacle à l'avancée de la cure en entravant la libre association et en conduisant parfois le sujet au silence, ce qui empêche le patient d'avoir accès aux désirs inconscients qui le déterminent partiellement, puisqu' "il existe une force qui les empêche de devenir conscient" (Freud, 1909). La résistance donc est l'effort que produit le "refoulement" pendant la cure, ce mécanisme de défense contrariant la remémoration et l'interprétation des souvenirs difficiles.

Identifier une résistance dans une image mentale, consiste à projeter entre les différents éléments constituant l'image un lien de causalité linéaire, à l'image d'un empilement d'objets en équilibre ou d'une rangée de dominos. Si bien que la moindre modification au sein de cette structure hiérarchisée, suite à l'apparition ou à l'évolution des données du projet entre deux images mentales successives, détruit l'équilibre statique du projet et nécessite de la part du concepteur d'en projeter une autre ( $c f$. figure 3).

De ce qui précède, au cours d'un processus de conception architecturale, nous posons que la résistance est le degré de persistance de la dimension statique du modèle architectural au cours d'une séquence d'images mentales du projet, la persistance partielle d'une représentation structurelle du projet d'architecture au cours du temps.

\footnotetext{
${ }^{20}$ Avec le mot résister, le mot résistance apparaît au XIII ${ }^{\mathrm{e}}$ siècle, venant du latin resistere (s'arrêter, résister), du verbe sistere (s'arrêter).
} 

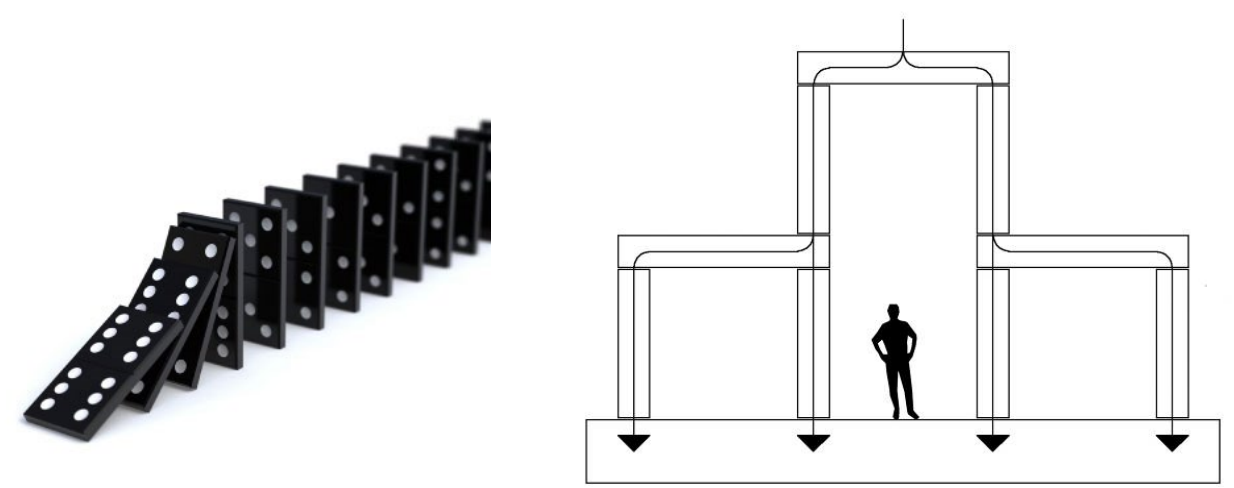

Fig. 3 - 1. Causalité linéaire au sein d'une série de dominos : si un domino tombe, tous les dominos tombent, l'ensemble de la structure hiérarchisée s'écroule. 2. Exemple de structure hiérarchisée verticalement (Claeys, 2017a).

\section{LA RÉSILIENCE DU MODÈLE DYNAMIQUE}

Devenu polysémique, le concept de résilience ${ }^{21}$ a été transposé, avec plus ou moins de succès, dans de nombreux domaines (psychologie, économie, écologie...). Bien que devenu fourre-tout, l'origine étymologique du mot est riche et, lorsqu'il est utilisé comme concept, il se décline de manière avantageuse dans plusieurs champs.

En physique des matériaux, la résilience a parfois désigné la capacité d'un objet à retrouver son état initial après un choc ou une pression continue. Ayant inventé une machine appelée le mouton-pendule, l'ingénieur français Georges Charpy a réalisé un essai célèbre (1901, cf. figure 4) : une pièce d'acier entaillée subit un choc visant à la rompre. La sollicitation menant à la rupture permet l'estimation de la "ténacité" de la pièce en mesurant l' "énergie de rupture". Cette expérience est alors répétée en variant la température de la pièce d'acier de manière à déduire son comportement. Plutôt que de ténacité, l'essai est régulièrement appelé l'essai de résilience Charpy. Dans cette acception, le concept de résilience est alors très proche de celui d'élasticité de la matière, bien que par comparaison au sens qui lui est donné aujourd'hui, il serait plus proche de celui de plasticité.

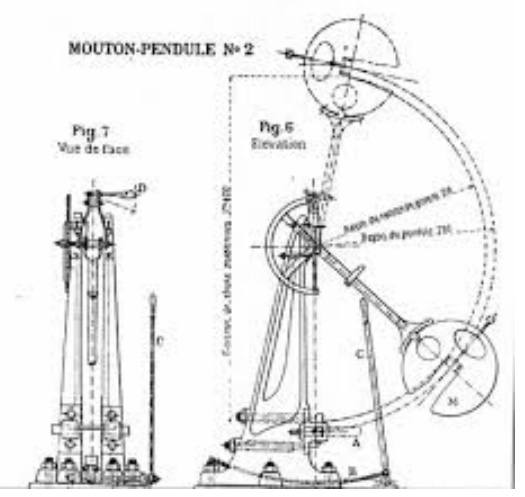

Fig. 4 - Georges Charpy, La machine mouton-pendule pour réaliser des essais de flexion par choc, 1904.

Lors de son passage de la physique aux sciences sociales, le concept a été popularisé notamment par le neuropsychiatre français Boris Cyrulnik (1999) et il signifie, selon le sociologue hollandais Stefan Vanistendael (1998, cité par Cyrulnik, 1999), "la capacité à réussir, à vivre et à se développer positivement, de manière socialement acceptable, en dépit du stress ou d'une adversité qui comporte normalement le risque grave d'une issue négative". La résilience désigne alors un processus

\footnotetext{
${ }^{21}$ Du latin resilio (rebondir) - composé des mots re (mouvement arrière) et salire (sauter, bondir) -, le mot résilience - apparu en anglais en 1824 et en français à la fin du XXe siècle - désigne en anglais - au moins depuis le XVII ${ }^{\mathrm{e}}$ siècle - reculer pour mieux sauter (resiliency).
} 
psychologique permettant à un sujet ayant subi un événement traumatique, ou souffrant de conditions de vie difficiles, de continuer à se projeter dans l'avenir. Alors que dans le cadre de l'essai de résilience Charpy, le matériau revient à son état initial après la sollicitation, en sciences sociales, le sujet passe d'un état d'équilibre à un autre, différent du précédent. De manière générale, la forme, la structure ou la fonction peuvent changer, seule la finalité projective du système est conservée.

À plusieurs reprises, Cyrulnik (2004) a proposé une interprétation personnelle de l'idée du psychologue et philosophe américain William James (1890) - l'un des fondateurs du pragmatisme - selon laquelle "la vie mentale est, avant tout, une finalité", avec pour premier objectif la conservation de l'individu, en racontant la fable des casseurs de cailloux, qu'il attribue avec humour à l'écrivain français Charles $\mathrm{P}$. Péguy ${ }^{22}$ : "En se rendant à Chartres, Péguy voit sur le bord de la route un homme qui casse des cailloux à grands coups de maillet. Son visage exprime le malheur et ses gestes la rage. Péguy s'arrête et demande : 'Monsieur, que faites-vous ?' 'Vous voyez bien, lui répond l'homme, je n'ai trouvé que ce métier stupide et douloureux.' Un peu plus loin, Péguy aperçoit un autre homme qui, lui aussi, casse des cailloux, mais son visage est calme et ses gestes harmonieux. 'Que faites-vous, monsieur ?', lui demande Péguy. 'Eh bien, je gagne ma vie grâce à ce métier fatiguant, mais qui a l'avantage d'être en plein air', lui répondit-il. Plus loin, un troisième casseur de cailloux irradie de bonheur. Il sourit en abattant la masse et regarde avec plaisir les éclats de pierre. 'Que faites-vous ?', lui demande Péguy. 'Moi, répond cet homme, je bâtis une cathédrale !'." Selon Cyrulnik: "Le caillou dépourvu de sens soumet le malheureux au réel, à l'immédiat qui ne donne rien d'autre à comprendre que le poids du maillet et la souffrance du coup", alors que "celui qui a une cathédrale en tête transfigure le caillou". Si bien que : "Le fait est le même, l'attribution du sens au fait est totalement différente. Et cette attribution du sens vient de notre propre histoire et de notre contexte social. Quand on a une cathédrale dans la tête, on ne casse pas les cailloux de la même manière."

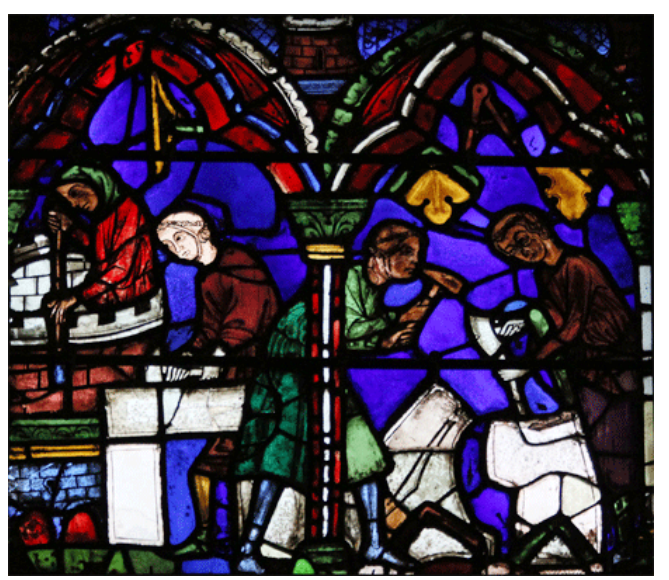

Fig. 5 - Maçons et tailleurs de pierre, $1^{\text {er }}$ panneau, vitrail de la vie de saint Chéron, cathédrale Notre-Dame de Chartres, XIII ${ }^{\mathrm{e}}$ siècle.

Par ailleurs, la résilience psychologique serait rendue possible par la "plasticité neuronale" de notre cerveau. L'histologiste espagnol Santiago Ramón y Cajal (1913-1914), pionnier de la théorie neuronale, initie et utilise sommairement cette idée de plasticité du cerveau. Mais le neurologue britannique Geoffrey Raisman (1969) semblerait être le premier à avoir établi objectivement la capacité du cerveau à créer des synapses à la suite d'une lésion chez l'adulte. Au début du XXI ${ }^{\mathrm{e}}$ siècle, la notion a été ensuite popularisée par le neuroscientifique américain d'origine autrichienne Eric R. Kandel (1999) attribuant aux neurosciences - et à la plasticité neuronale - un potentiel énorme pour les recherches à venir ${ }^{23}$.

\footnotetext{
${ }^{22}$ Certaines œuvres de Charles P. Péguy sont d'inspiration médiévale et il aurait fait deux pèlerinages jusqu'à Chartres, en trois jours de marche, en 1912 et en 1913, mais il n'aurait jamais écrit cette fable.

${ }^{23}$ Par exemple, une étude en IRM réalisée chez des chauffeurs de taxi a montré que les zones du cerveau qui contrôlent la représentation de l'espace sont plus développées, et ce proportionnellement au nombre d'années d'expérience de la conduite du taxi (Maguire et al., 2000).
} 
Appliqué à l'individu, le concept a été élargi à la société en tant que groupe d'individus, assorti d'une identité collective. La société ne possède pas une résilience égale à la simple somme des résiliences individuelles de ses membres. En tant que système résultant des relations entre plusieurs individualités capables de résilience psychologique, la société en développe des modalités spécifiques.

Des sciences sociales, le concept a été utilisé par modéliser les écosystèmes. Après la survenue d'un aléa, la capacité d'un écosystème à se reconstituer est sa capacité de résilience (par exemple, par la diversité biologique, c'est-à-dire un nombre élevé d'espèces ou une grande diversité génétique au sein des espèces). Les chercheurs proposent de remplacer une gestion top-down (descendante) des écosystèmes, reposant sur des processus linéaires d'optimisation ayant montré leurs limites, par des modèles prenant en compte la complexité des dynamiques écologiques et sociétales. Ce type d'approche mène à l'analyse systémique des systèmes écosociaux. Parmi d'autres possibles, en promouvant la capacité de résilience des écosystèmes (Holling, 1973), deux analogies, plus descriptives qu'explicatives, sont régulièrement utilisées : les "cycles adaptatifs", représentant la trajectoire d'un écosystème en quatre phases, et la "panarchie", permettant la mise en relation d'un cycle avec d'autres niveaux d'organisation (Gunderson \& Holling, 2002, $c f$. figure 6).

En urbanisme et aménagement du territoire, des situations complexes sont modélisée à partir du concept de "métabolisme urbain", dont l'usage a été initié par les recherches en écologie industrielle (Wolman, 1965), ayant elles-mêmes contribué au développement de l'écologie territoriale (Barles, 2010), mais également de l'écologie sociale (Fischer-Kowalski \& Erb, 2016), fondée - entre autres - sur la théorie des systèmes sociaux de Niklas Luhmann (1984) et sur la théorie autopoïétique d'Humberto R. Maturana et de Francisco J. Varela (1972). Dans ces recherches métabolistes - qui doivent être distinguées du métabolisme japonais des années 1960 -, les méthodes de modélisation des écosystèmes et des sociétés se rejoignent, pour proposer des villes résilientes, capables d'absorber des perturbations en intégrant la complexité de l'évolution des réservoirs et des flux de matière, d'énergie et d'information traversant la ville, avec un objectif général de durabilité.
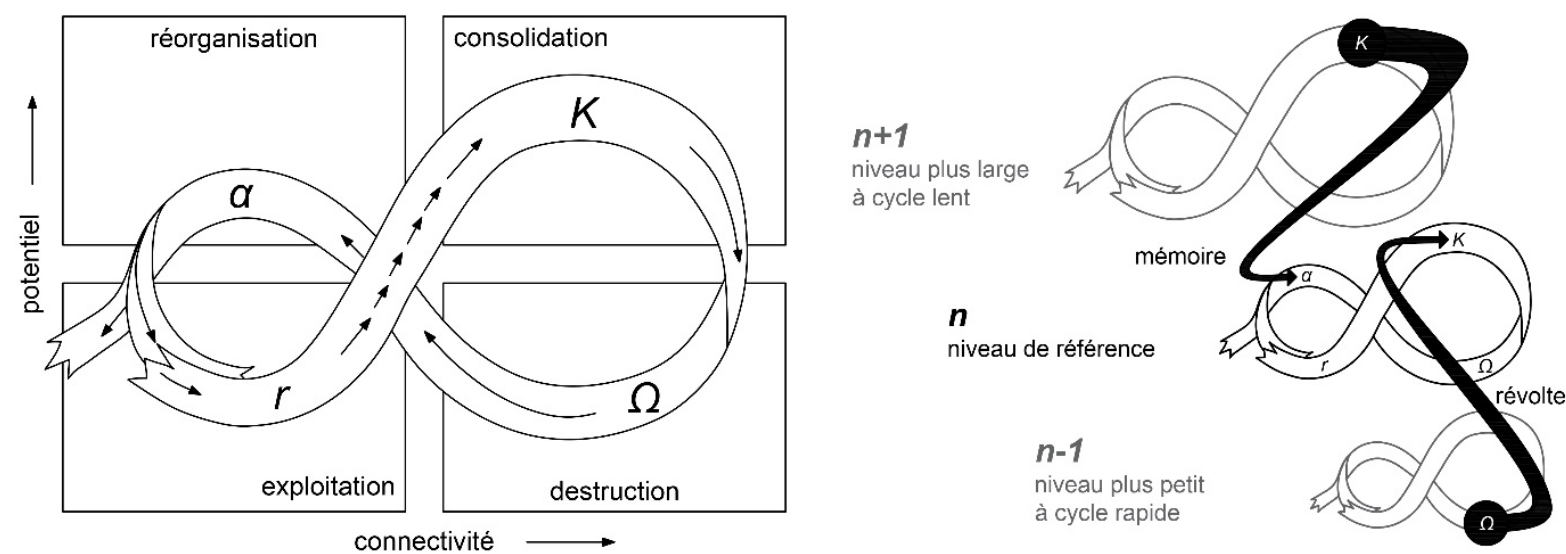

Fig. 6 - 1. Le cycle adaptatif. 2. La panarchie.

Schémas adaptés d'après Gunderson \& Holling (2002, p.34 et p.75).

À priori, les bâtiments ne sont pas plastiques ou élastiques. Traditionnellement, les édifices sont construits de manière pérenne, pour abriter leurs habitants de la pluie, du vent, du froid et des prédateurs. Plus encore, ils créent les conditions matérielles leurs permettant d'acquérir une "assise existentielle" (Norberg-Schulz, 1974). La seule altération prise en compte semble être l'usure du temps et la patine des matériaux. 
Pourtant, du point de vue de l'étude des structures constructives, une analogie évidente peut être opérées entre le concept de résilience et celui de "tenségrité" ${ }^{24}$, développé par l'architecte américain Richard Buckminster Fuller, notamment dans ses dômes géodésiques. En effet, lors de toute sollicitation de la structure en un point, les efforts sont répartis dans l'ensemble de la structure qui se déforme pour les reprendre. Outre les modélisations structurelles, d'autres projets nécessitent de la part du concepteur d'envisager la dimension dynamique du modèle architectural. Certains exemples emblématiques de l'histoire de l'architecture peuvent en témoigner : par exemple, la conception de projets de papier, tels que le Fun Palace à Londres (années 1960), conçu par Cedric Price et Joan Littlewood, ou la Walking City (1968), dessinée par Ron Herron pour Archigram, mais également la mise au point de prototypes tel que l'Aegis Hypo-Surface à Birmingham (1999) de deCOI Architects. Par ailleurs, ce type de modélisation dynamique des projets mène à de nombreuses analogies systémiques, dépassant le simple aménagement de l'environnement construit : "Les structures en tenségrité sont mécaniquement efficaces et elles donnent sens à notre époque : une société dynamique de quasi-sujets inter-reliés et sur-informés à la recherche de structures soutenables et économiques en matière et en énergie. Une société de flux migratoires à la recherche d'habitats modulaires et facilement constructibles et accessibles." (Claeys, 2017a)
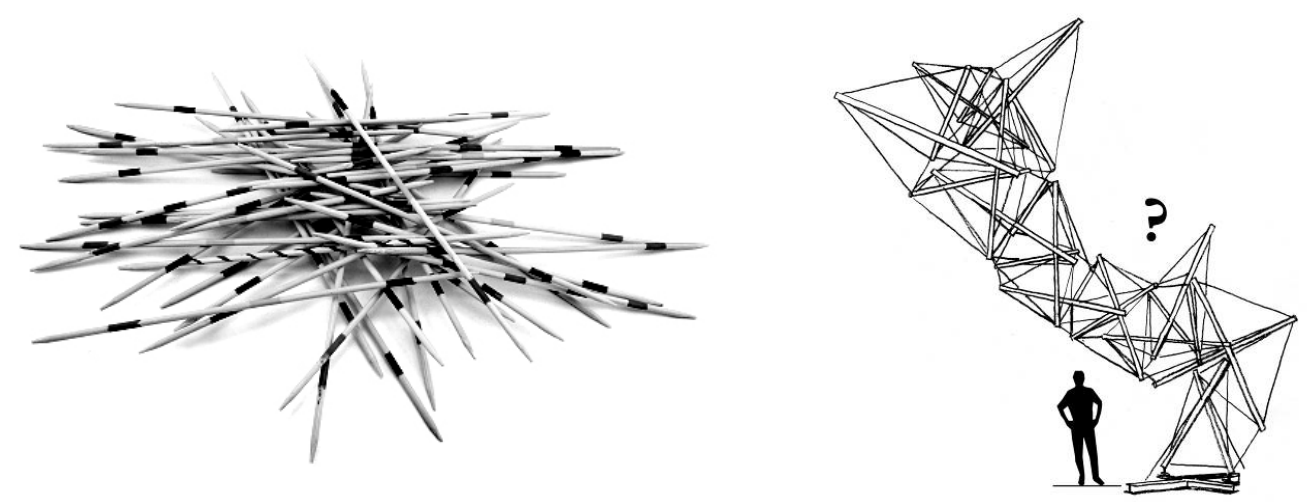

Figure 7 - 1. Causalité circulaire au sien d'une série de bâtons de mikado. Si l'un des bâtons bouge, tous les autres peuvent bouger, l'ensemble du système dynamique se reconfigure. 2. Exemple de structure relationnelle en tenségrité (Claeys, 2017a).

De manière générale, la résilience est la capacité d'un système écosocial à s'auto-organiser pour faire face à un aléa (interne ou externe), retrouver un équilibre et perdurer. Dans un système modélisé avec un degré de résilience, le changement pousse le modélisateur à utiliser le potentiel créatif du système, en laissant exercer les capacités d'apprentissage et d'adaptation des membres qui le constituent : l'aléa est accueilli de manière positive et la modélisation est dynamique, inclusive et participative. En fonction de la vulnérabilité irréductible (complexité, connectivité...), des besoins (ressources nécessaires) et des objectifs (finalité projective) que le modélisateur du système lui prête, la résilience est la capacité de celui-ci à absorber les perturbations pour conserver un équilibre. Si nécessaire, le système se réorganise, sans perdre son identité, en expérimentant des changements, tout en maintenant ses fonctions vitales et ses capacités de contrôle.

De ce qui précède, au cours d'un processus de conception architecturale, nous posons que la résilience est le degré de persistance de la dimension dynamique du modèle architectural au cours d'une séquence d'images mentales du projet, la persistance partielle d'une représentation fonctionnelle du projet d'architecture au cours du temps.

\footnotetext{
${ }^{24}$ Dans le brevet qu'il dépose, Richard Buckminster Fuller (1962) parle de "tensional integrity" (intégrité tensionnelle), ce qui a donné par contraction le mot tensegrity en anglais et, finalement, tenségrité en français. Il montre qu'une structure en tenségrité génère sa propre force tensionnelle (tensile) pour déployer une intégrité architecturale indépendante de la gravité (integrity). En maintenant des "small islands [of compression] in a sea of tension", une structure en tenségrité est donc "an assemblage of tension and compression components arranged in a discontinuous compression system".
} 


\section{CONCLUSION : D'UN ÉQUILIBRE À L'AUTRE}

Après avoir forgé les concepts de rémanence, de résistance et de résilience, une discussion doit encore avoir lieu: ces trois concepts transdisciplinaires sont-ils opérants pour expliciter partiellement les changements d'état en conception architecturale? Autrement dit, peuvent-ils apporter un éclairage sur le caractère évolutif de l'image mentale du projet d'architecture dans l'espace mental de conception ?

D'abord, d'un point de vue heuristique, les trois concepts interviennent dans la mise en relation des opérations de conception et des opérandes, en manifestant une forme de permanence de ces derniers. En effet, au cours du processus de conception, ces concepts (rémanence, résistance, résilience) participent à la détermination des poids associés aux opérandes dans les trois plans du modèle mental du projet (perceptif, statique, dynamique). Ils traduisent l'évaluation subjective, opérée par le concepteur, du degré de persistance de ces opérandes, par rapport aux opérations de conception menées sur elles (composition, construction, régulation) :

- la rémanence est le degré de persistance du plan perceptif du modèle architectural au cours du temps, la permanence partielle de la représentation formelle du projet d'architecture au sein d'une séquence d'images mentales du projet, affectée par une succession d'opérations de composition;

- la résistance est le degré de persistance du plan statique du modèle architectural au cours du temps, la permanence partielle de la représentation structurelle du projet d'architecture au sein d'une séquence d'images mentales du projet, affectée par une succession d'opérations de construction;

- la résilience est le degré de persistance du plan dynamique du modèle architectural au cours du temps, la permanence partielle de la représentation fonctionnelle du projet d'architecture au sein d'une séquence d'images mentales du projet, affectée par une succession d'opérations de régulation.

Ensuite, du point de vue des champs d'applications de la discipline, en partant de l'hypothèse que tout processus de conception architecturale est d'abord la construction d'une image mentale évolutive, sous la forme d'un projet d'architecture, la dimension neuropsychologique associée au fonctionnement cognitif du concepteur devrait toujours être prise en compte dès le départ. Il faudrait étudier la psychologie du concepteur à un niveau méta pour appréhender l'architecture, avant de l'étudier dans ses différents champs d'application (architecture, ingénierie architecturale, urbanisme, patrimoine, paysage...). Ainsi, bien que leur transposition potentielle devrait encore être fortement étudiée, les trois concepts étudiés ici seraient suffisamment généraux pour être déclinés de manière transdisciplinaire, à la fois, au niveau neuropsychologique de la conception et au niveau de différentes applications disciplinaires ( $c f$. tableau 1).

Enfin, du point de vue de la pratique de la conception architecturale, ces trois concepts ouvrent indirectement une réflexion plus globale. À l'ère de l'information, le concepteur est, à la fois, "immergé", "externalisé" et "en réseau" (Claeys, 2019). Il est confronté à une quantité exponentielle d'informations, dont l'origine des mises en relation est difficile à déterminer. Le dynamisme extrême des échanges intempestifs de données forme un insondable bruit, rendant toute prise de décision difficile pour le concepteur. Or, la société civile attend de ce dernier qu'il ait l'habilité à opérer une synthèse à partir de données variées, appartenant à des champs disciplinaires éparses. Autrement dit, la capacité à équilibrer, en cours de processus, de multiples contraintes pour concevoir l'organisation et la description complète d'un projet, matérialisable dans l'environnement construit, sous la forme d'un artefact - un édifice permettant la réalisation de potentialités inhérentes au réel. Cette situation illustre l'omniprésence d'un quatrième concept : celui d'équilibre (équilibre du modèle architectural, équilibre perceptif, statique ou dynamique...). En effet, pour opérer une synthèse, une des principales capacités cognitives du concepteur est de rééquilibrer constamment les différents plans du modèle mental du projet en fonction d'une finalité projective, d'identifier la redondance dans le magma informationnel qui l'entoure, d'établir subjectivement les parties du projet qui persistent, d'un état à l'autre du processus, pour en tirer les informations jugées pertinentes. 
Les concepts de rémanence, résistance et résilience aident le concepteur à endosser réflexivement l'indétermination, à assumer la subjectivité nécessaire au choix des données prises en compte pour concevoir le projet. Confronté à l'immédiateté d'accès et à l'apparente exhaustivité des données disponibles, ils l'aident à pondérer et équilibrer la persistance des parties du modèle mental du projet en cours.

\begin{tabular}{l|l|l|l}
\hline & $\begin{array}{l}\text { rémanence } \\
\text { (plan perceptif) }\end{array}$ & $\begin{array}{l}\text { résistance } \\
\text { (plan statique) }\end{array}$ & $\begin{array}{l}\text { résilience } \\
\text { (plan dynamique) }\end{array}$ \\
\hline neurologie & inscription neuronale & rigidité neuronale & plasticité neuronale \\
\hline psychologie & $\begin{array}{l}\text { perception } \\
\text { (perception de phénomènes) }\end{array}$ & $\begin{array}{l}\text { résistance } \\
\text { (refoulement d'informations) }\end{array}$ & $\begin{array}{l}\text { résilience } \\
\text { (réorganisation d'informations) }\end{array}$ \\
\hline $\begin{array}{l}\text { conception } \\
\text { architecturale }\end{array}$ & opération de composition & opération de construction & opération de régulation \\
\hline $\begin{array}{l}\text { conception } \\
\text { métabolique }\end{array}$ & $\begin{array}{l}\text { analyse typomorphologique du } \\
\text { territoire urbain pour en dégager } \\
\text { les éléments permettant de le } \\
\text { modéliser (types, formes, } \\
\text { réservoirs et flux de matière, } \\
\text { d'énergie et d'information) }\end{array}$ & $\begin{array}{l}\text { modélisation du territoire urbain } \\
\text { àl'aide de structures écosociales } \\
\text { fermées, pour limiter des risques } \\
\text { associés négativement à des } \\
\text { effets exogènes, en empêchant } \\
\text { tout changement non désiré }\end{array}$ & $\begin{array}{l}\text { modélisation du territoire urbain } \\
\text { àide de systèmes écosociaux } \\
\text { ouverts, pour intégrer des effets } \\
\text { positifs de l'évolution du } \\
\text { territoire }\end{array}$ \\
\hline $\begin{array}{l}\text { conception } \\
\text { patrimoniale }\end{array}$ & $\begin{array}{l}\text { identification } \\
\text { (relever des préexistences à l'état }\end{array}$ \\
$\begin{array}{l}\text { actuel pour en établir une lecture } \\
\text { descriptive) }\end{array}$ & $\begin{array}{l}\text { restitution } \\
\text { (rétablir l'édifice de manière } \\
\text { figée, uniquement dans un état } \\
\text { supposé original) }\end{array}$ & $\begin{array}{l}\text { réhabilitation } \\
\text { (redonner une destination à } \\
\text { l'édifice tout en gardant ses } \\
\text { valeur patrimoniale) }\end{array}$ \\
\hline
\end{tabular}

Table 1 - Première hypothèse d'opérabilité des concepts de rémanence, de résistance et de résilience dans différents champs d'application de la conception architecturale.

\section{RÉFÉRENCES}

BARLES, Sabine (2010). "Society, Energy and Materials: What are the Contributions of Industrial Ecology, Territorial Ecology and Urban Metabolism to Sustainable Urban Development Issues ?". Journal of Environmental Planning and Management, 53(4), pp.439-455.

CHARPY, Georges (1901). "Note sur l'essai des métaux à la flexion par choc de barreaux entaillés". Mémoires et comptes rendu de la société des ingénieurs civils de France, pp.848-877.

CLAEYS, Damien (2020). "Discontinuities in representation processes: The case of architectural design". Keynote speaker, 16th HSSS National \& International Conference, Tripoli, Grèce (2426/09/2020).

CLAEYS, Damien (2019). "Trois figures architecturales post 1969 : Les concepteurs immergé, externalisé et en réseau". lieuxdits, UCL-LOCI, n¹7, décembre, pp.25-35.

CLAEYS, Damien (2018). "Discontinuités des processus de conception architecturale : Crises de la représentation ?". Acta Europeana Systemica (AES), n8, pp.19-36.

CLAEYS, Damien (2017b). "De l'interprétation créative du réel au processus bayésien de conception architecturale". Acta Europeana Systemica (AES), n7, pp.65-80.

CLAEYS, Damien (2017a). "La conception de structures en tenségrité : Analogies systémiques". lieuxdits, UCL-LOCI, n²12, avril, pp.22-27.

CLAEYS, Damien (2016). "Le fantasme du démiurge : L'architecte soumis à la tentation du pouvoir". Acta Europeana Systemica (AES), n6, 14pp.

CLAEYS, Damien (2015). "Concevoir un projet d'architecture: Calmer les certitudes, gérer l'incertitude". Lieuxdits : Sérendipité, UCL-LOCI, n9, avril, pp.20-23. 
CLAEYS, Damien (2013). Architecture \& complexité : Un modèle systémique du processus de (co)conception qui vise l'architecture. Thèse de doctorat de l'Université catholique de Louvain. Louvain-la-Neuve : Presses universitaires de Louvain, 445pp.

CYRULNIK, Boris (2004). Parler d'amour au bord du gouffre. Paris : Odile Jacob, éd.2007.

CYRULNIK, Boris (1999). Un merveilleux malheur. Paris : Odile Jacob, éd.2002.

FELTZ, Bernard (2003). La science et le vivant: Introduction à la philosophie des sciences de la vie. Bruxelles : De Boeck \& Larcier.

FREUD, Sigmund (1909). Cinq leçons sur la psychanalyse. Trad. Y. Le Lay [Ueber Psychoanalyse, fünf Vorlesungen]. Paris : Payot, éd.2001.

FULLER, Richard Buckminster (1962). Tensile-Integrity Structures. U.S. Patent No 3.063.521 A, 13 novembre 1962.

GUNDERSON, Lance H. \& HOLLING C. S. (2002). Panarchy: Understanding Transformations in Human and Natural Systems. Washington: Island Press.

HOLLING, C. S. (1973). "Resilience and stability of ecological systems". Annual Review of ecology and systematics, $\mathrm{n}^{\circ} 4,23 \mathrm{pp}$.

JAMES, William (1908). The Principles of Psychology. New York: Henry Holt and Company, 2 vol.

KANDEL, Eric R. (1999). "A new intellectual framework for psychiatry". American journal of psychiatry, Vol.155, Issue ${ }^{\circ} 4, \mathrm{pp} .457-469$.

LE MOIGNE, Jean-Louis (1990). La modélisation des systèmes complexes. Paris : Dunod, éd.1999.

LUHMANN, Niklas (1984). Systèmes sociaux : Esquisse d'une théorie générale. Trad. Lukas K. Sosoe [Soziale Systeme: Grundriß einer allgemeinen Theorie. Berlin: Suhrkamp Verlag]. Québec : Presses de l'Université Laval, éd.2010.

MAGUIRE, Eleanor A., GADIAN, David G., JOHNSRUDE, Ingrid S., GOOD Catriona D., ASHBURNER, John, FRACKOWIAK, Richard S.J. \& FRITH, Christopher D. (2000). "Navigationrelated structural change in the hippocampi of taxi drivers". Proceedings of the National Academy of Sciences of the United States of America, vol.97, n 8, pp.4398-4403.

MATURANA, Humberto R. \& VARELA, Francisco J. (1972). "Autopoiesis: The Organization of the Living". Trad. Maturana, H.R. [De Máquinas y Seres Vivos]. Autopoiesis and Cognition: The Realization of the Living. Dordrecht: Kluwer Academic Publishers, éd.1980, pp.59-138.

MORIN, Edgar (1986). La méthode 3 : La connaissance de la connaissance. Paris : Seuil (coll. Points). NACCACHE, Lionel (2020). Le cinéma intérieur : Projection privée au cœur de la conscience. Paris : Odile Jacob (coll. Sciences).

NORBERG-SCHULZ, Christian (1974). La signification dans l'architecture occidentale. Trad. A. M. de Dominicis [Significato nell'architettura occidentale. Milano: Electa]. Liège/Bruxelles : Mardaga, éd.1977.

PURVES, Dale, PAYDARFAR, Joseph A. \& ANDREWS, Timothy J. (1996). "The wagon wheel illusion in movies and reality". Proceedings of the National Academy of Sciences of the United States of America, vol. 93, issue 8, pp.3693-3697.

RAISMAN, Geoffrey (1969). "Neuronal plasticity in the septal nuclei of the adult rat". Brain research, vol.14, issue 1, pp.25-48.

RAMÓN Y CAJAL, Santiago (1913-1914). Estudios sobre la degeneración y regeneración del sistema nervioso. Madrid: Imprenta de Hijos de Nicolàs Moya.

ROSNAY, Joel (de) (1975). Le Macroscope : Vers une vision globale. Paris : Seuil (coll. Points). STEIWER, Jacques (2010). Vers une théorie de la connaissance systémique. Paris : L'Harmattan. VANISTENDAEL, Stefan (1998). Clés pour devenir : la résilience. Les Vendredis de Châteauvallon, nov.1998, Genève. 
VANRULLEN, Rufin, REDDY, Leila \& KOCH, Christof. (2006). "The continuous wagon wheel illusion is associated with changes in electroencephalogram power at approximately $13 \mathrm{~Hz}$ ". Journal of Neuroscience, vol.26, issue 2, pp.502-507.

WATZLAWICK, Paul (1978). La réalité de la réalité : Confusion, désinformation, communication. Paris : Seuil (coll. Points).

WOLMAN, Abel (1965). "The Metabolism of Cities". Scientific American, Vol.213, pp.179-190. 